\title{
iFREEPHONY: MINIMALISTIC INTERFACE DEVELOPED FOR THE VISUALLY IMPAIRED AND THE ELDERLY COMMUNITY IN BANGLADESH
}

\author{
Nova Ahmed, Fajilatun Nahar \& A. M. Masudul Haque \\ Department of Electrical and Computer Engineering \\ North South University, Bangladesh
}

nova.ahmed@northsouth.edu; rupafn@gmail.com; saadixl@gmail.com

\begin{abstract}
Touchscreen-based phones can be challenging for people with visual impairment. These phones require special features to cater to their needs when it comes to a developing country's perspective like Bangladesh. The interface needs to remain simple as well as it must support the required functionalities. It is often observed that touchscreen phones are not desirable for the user community in Bangladesh as it offers a complex set of functions. We have considered visually impaired personnel in terms of physical limitations and the community who falls in the older age limit as our target community. Our user study has confirmed that the visually impaired user community here asks for a simple and functional interface. We have designed, developed, and deployed a simple system ifreePhony that captures the minimalistic features. We have considered both the elderly and the visually impaired community in our user studies. It shows around 36\% reduction in search time and around 57\% increase in text typing compared to a traditional phone.
\end{abstract}

Keywords: Touchscreen interface, accessible interface, minimalistic touch screen features, smart phone in Bangladesh.

\section{INTRODUCTION}

A large number of people are not able to take advantage of the recent advancement in mobile computing. The situation exacerbates when this population includes people who require specialized attention e.g. people with 
visual impairment and the elderly population. Smart phones are designed to make lives easier by opening up many new avenues through software and intuitive usage. However, the situation is different for a group of population who are partially or fully visually impaired in a developing country like Bangladesh. The concept of user centric design is emerging but it has not met the unique needs of population with special needs. Although there are a variety of tools available for the visually impaired people, none of them is being used by them in Bangladesh. We have worked closely with them to design a system along with its usable interface that would meet their unique needs, and have designed, implemented and tested a system that is simple enough for general usage, along with a complete set of required functionality.

There is an array of work focusing on mobile phone interface, hardware and gesture detection as presented by Kim, Bessho, Kobayashi, Koshizuka, \& Sakamura, (2016); Ghilardi, Junior, \& Manssour, (2016); Kvetny, Kahn, Cohen, \& Carmel, (2016); Buzzi, Buzzi, Leporini, \& Trujillo, (2014); Raposo, Rios, Lima, Gadelha, \& Castro, (2014); Oh, Kane, \& Findlater, (2013); and McGookin, Brewster, \& Jiang, (2008) - They all focused on the usage by visually impaired people. Our work does not try to provide the largest number of features; rather, it focuses on the minimal number of features required by a group of visually-impaired people in Dhaka, Bangladesh. They feel overburdened when various features are present. We discuss the related work in detail later.

We have considered a simple interface for the target population of visuallyimpaired personnel and elderly personnel in the context of Bangladesh, setting the stage for developing country requirements. We have worked with a group of visually-impaired people in an urban setup who are pursuing their studies and are open to try new tools to enhance their lives. These users are willing to use a smart phone to take advantage of the positioning-and locality-based tools. On the other hand, the open indistinguishable touch interface often makes the system difficult to use. We have focused, in our design, simplicity and ease of use. We have limited the features to the minimal level as suggested by the possible users. The openness of the smart phone screen appears to this group of users as a challenge. Searching and emergency calls are mostly done by memorization and often by text to speech software which can be easily adopted in a complete system. Our user study was done in an urban area of Bangladesh where we had participants who were mostly students in various institutes, who used cell phones regularly in their daily operations. We developed a system, iFreePhony, which tries to meet the unique minimal requirements of developing countries' perspectives. 
We extended the research presented by Ahmed, N., Nahar, F., Haque A. M. M., and Chowdhury, A. M. (2015) we conducted a usability study of the finished system to find out the strengths and limitations of iFreePhony and compared the performance of searching and typing using our system and the existing system without any custom application. Our contribution can be specified as follows: first we provide user requirement evaluation from a developing country, specifically, Bangladesh; second, we focuse on designing and implementing the system suggested by the user group; and finally, we presented the work in the deployment of the system along with testing for further improvement and user feedback.

The rest of the paper is organized in the following way; we discuss the related work in section two, followed by user requirements, user interface and implementation. We describe the solution approach and conclude the work in the given sequence.

\section{RELATED WORK}

Touchscreen interfaces have become VERY popular over the last few years. A number of assistive technology is being developed nowadays as a result of the proliferation of smart operating systems and their application compatibility. The accuracy of such technologies is increasing rapidly with increasing computational performance.

During our project we reviewed a number of paper and professional applications related to our work directly and indirectly. Though most of the related projects worked with different user interface with different functionality for visually impaired people using speech synthesis and other gestures, but we mainly focused on the most important feature that is the phone call. All the papers as presented by Buzzi et al. (2014), Raposo et al. (2014) and McGookin et al. (2008) are mainly concerned about using the touch screen devices to assist the visually impaired people in different ways. Gestures like swiping, tapping etc. has been talked about in those papers. One of the most popular tools for the visually-impaired is based on touchscreen phone is assisting in navigation. Buzzi et al. (2014) talks about assisting the navigation of a visually-impaired user through supporting voice commands, talk back and a special map for navigation. The application allows the user to ask the phone about their location; the application processes the voice input of the user and acts according to the commands as well as allows the user to choose the source and the destination point from the map. It will talk about the nearby places along the path. And the 
most interesting feature is the application will make the phone vibrate when the user is going in a wrong direction. Our system, on the other hand, provides a simpler set of features that will be usable in their day-to-day lives.

The recent interests in research work suggest the emerging usage of smart phones to support the visually-impaired population. The work presente by Kim et al. (2016) presents a voice-interface-based system for visually-impaired people as a support in train stations. Ghilardi et al. (2016) considers an imageprocessing-based system to support navigation. Kvetny et al. (2016) presents ideas on supporting navigation support for the visually-impaired people. However, the sophisticated navigation system is not one of the most desirable features in the smartphones of Bangladesh due to the high cost of Internet, low frequency of GPS locations along with poor support for infrastructure (roads, stations etc.). Raposo et al. (2014) present a multimodal keyboard on a simple dialer pad based on touch gesture, vibration, sound and speech. Similarly, the paper presented by McGookin et al. (2008) talks about different types of touch screen devices and their compatibility for the visually-impaired users. Niazi, Khusro, Khan, \& Alam, (2016) discusses a convenient layout of keyboards that are easier to type SMS messages by the visually-impaired personnel. Our work focuses more on a phone application rather than a specific feature of hardware to minimize the cost. Our work particularly differs from work that discuss generic usability metrics to evaluate mobile phone performances as conducted by Hussain, Hashim, Nordin, \& Tahir, (2013). Here, a requirement capture study was conducted by the students of the University of Glasgow. They investigated ways of overcoming touchscreen accessibility problems by comparing a raised paper overlay touchscreen-based MP3 player with a touchscreen gesture-based player. Another study was conducted by the students of the University of Maryland supported by Oh et al. (2013) that proposed the use of a text-to-speech technology and gesture sonification to generate sound based on finger touches. Our work can be considered to be a subset of this work looking at the minimalistic features that are asked for.

There are commercial and available applications that are used by the visuallyimpaired community in Bangladesh such as the JAWS Screen Reader (2016), iOS Voiceover (2016),Google Talkback (2016), TapBeats (2015) and Ariadne GPS as presented by Ariandne (2016). All the mentioned tools are utility software designed to improve user experiences but not specifically designed for the visually-impaired personnel. However, it was important to find out how these tools have been used to support the visually-impaired community in Bangladesh which has been a major source of motivation to design a complete tool to support the community in Bangladesh. 
There are interesting sets of work evolving around, the needs of developing and under-developed countries using the novel mobile-based applications presented by Dell, Francis, Sheppard, Simbi, \& Borriello (2014); DeRenzi, Borriello, Jackson, Kumar, Parikh, Virk, \& Lesh (2011); Larson, Goel, Boriello, Heltshe, Rosenfeld, \& Patel, (2012); Hartung, Lerer, Anokwa, Tseng, Brunette, \& Borriello, (2010); Haque et al., (2014), and Yasir et al. (2014). One major application of the mobile phone-based applications in-developing country context is healthcare and many researchers have taken the advantage to explore that aspect with novel ideas supported by Dell et al. (2014), DeRenzi et al. (2011), and Hartung et al. (2010) have explored innovative ways of using the mobile phone to support the information system-based application. The research work conducted by Yasir, Nibir, \& Ahmed (2014) uses the mobile phone to improve the healthcare situation in Bangladesh which shows the importance of the mobile phone-based application in the context of this country. Each and every work mentioned here focuses on various aspects of the novel mobile device usage but are not focused on the group of people we have considered.

It is interesting to find an array of work focused on visually-impaired people which would lead to wider availability of products in future.

\section{USER REQUIREMENTS}

We were fortunate to get the support from the Bangladesh Visually Impaired Peoples Society (2015) located at Dhaka, Bangladesh. We contacted them over the phone and they welcomed the research initiative gladly and spent almost an entire day discussing their challenges.

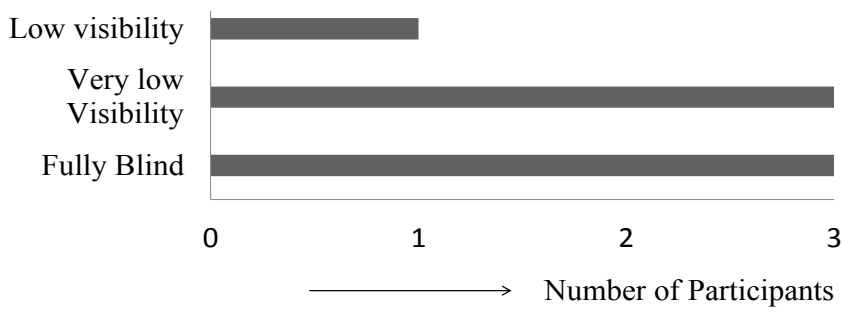

Figure 1. Use of assistive technology on phone. 


\section{Demography and Visibility Conditions}

We were accompanied by 7 individuals -2 females and 5 males; all in the range of 20 to 35 years. Most of them were students (71\%) and the rest were unemployed. We had three fully blind people - born with blindness; one had blurry and the other three had limited visibility (shown in Figure 1) as they mentioned: Everything appears very dark and blurry.

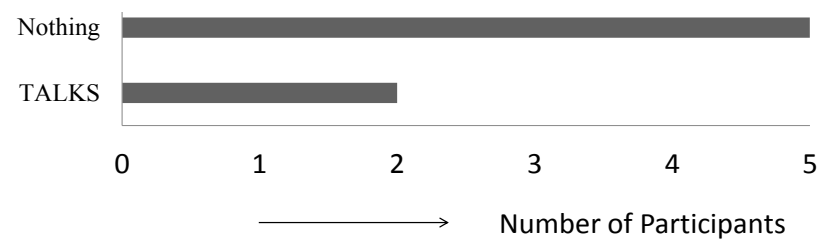

Figure 2. Use of assistive technology on phone.

\section{Cell Phone Usage and Experiences}

All the 7 participants used mobile phones actively -3 of them used the Nokia Symbian phone and the rest (4) used the feature phones with buttons. Their major cell phone usage included making telephone calls and often sending text messages as shown in Figure 2. We discovered that three of them used Nokia Symbian phones which had buttons and the other four of them used feature phones with buttons like symphony feature phones. And all of them had experiences with touch screen devices. We asked the interviewees why they used their phones. Three of them answered that they used their phones for only calling and texting. Four of them said they also used their phones for listening to music and radio.

We asked them if they used any assistive technology on their phones. Two of them used a software called TALKS on their Nokia Symbian phones which was is basically a straight forward screen reader.

\section{Desired Features in a Phone}

The majority (57\%) of our participants mentioned about phone book contact reader; $28 \%$ of the participants considered the Internet, better talk back and caller id alert as features they would ask for. The major concerns are presented in Figure 3. Individuals reported a lack of missed calsl alert and small texts to be their concerns. 
It would be best for us if we can hear the names of the contacts while browsing the phonebook.

Some people used a screen reader called TALKS. The screen readers I've used so far are very problematic sometimes. The speech synthesized voice is very robotic and sometimes confusing because it reads wherever I touch.

Some participants mentioned about the emergency situation support. All our interviewees said that they sometimes faced this problem as mentioned:

I sometimes get lost and do not understand where I am and have to rely on the kindness of others to take me back home.

Our participants have considered looking at smart phones and have faced major challenges.

I tried touchscreen phone once but it was horrible. I tried to tap something and it was mis-tapped. Mistakenly it opened another option.

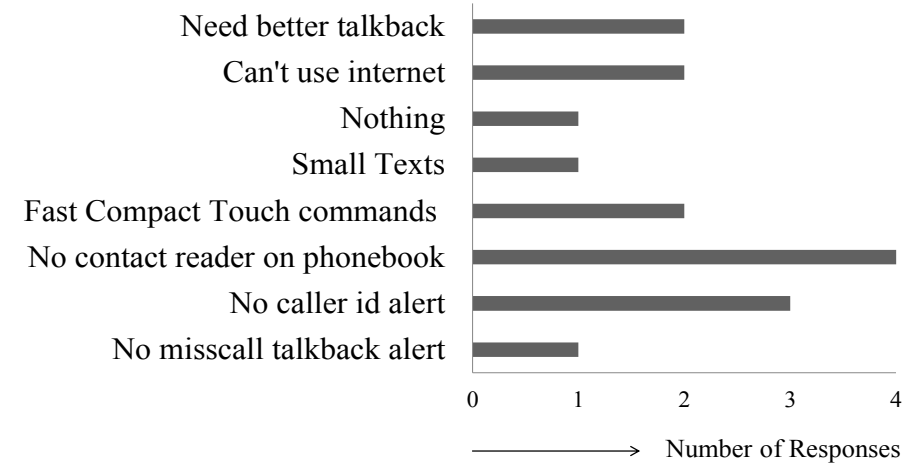

Figure 3. Problems of using phones.

We explained our initial plan of the application and asked them what part they liked and what their suggestion were about this application. Three of them said that they liked our screen reading and selection. Two of them said that they liked how we were planning to develop the phonebook for them. And most importantly all them liked the emergency contact option of the application. We asked their opinions about modifications or any suggestions for our application. They said that we should make sure that the application was fully free of cost. They also mentioned that we should keep the texts bigger and keep a sequential navigation if possible. 
After the analysis of all the survey results and the interviews, we figured out the problems of using the phone as well as the touch screen phones as summarized in Figure 4. Among them we chose two problems to solve. One was making them able to make a call in general and make contact in emergency situations. The other was browsing the phonebook. Also we solved the smaller text problem for people with lower vision.

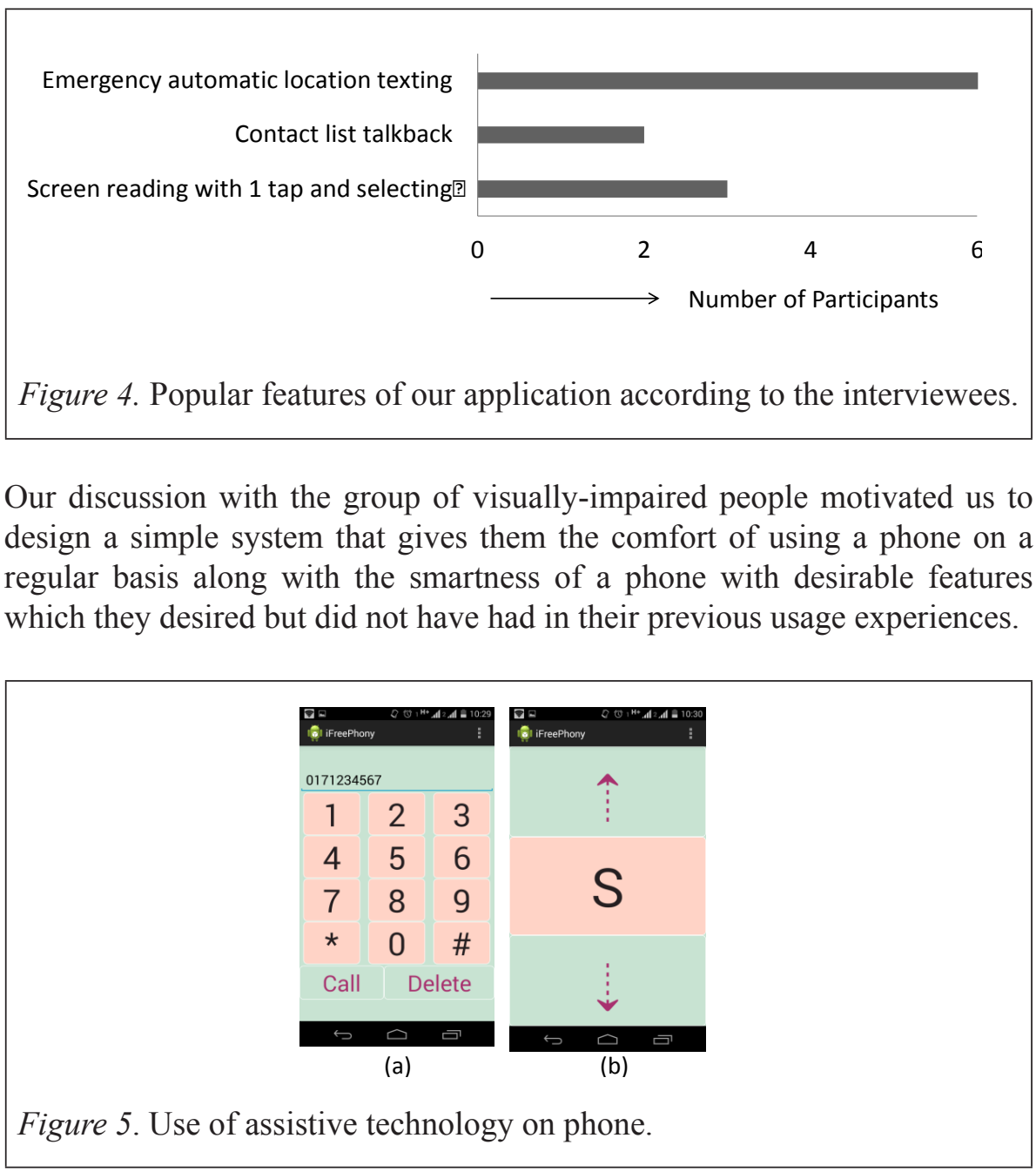

\section{USER INTERFACE}

Based on the problems and suggestions from our survey we designed and implemented a user interface with four major features: custom dialer, custom 
phone book, easy way to search contacts and an option to check for the current location and send emergency text messages.

Custom dialer: We included a custom dialer for typing numbers and making phone calls. This dialer has a clip board on the top as shown in Figure 6(a) where there is a sample number. There are big digits to type numbers, and in the bottom there are two buttons. In this portion as well as everywhere in the application we used two gestural pattern for screen reading and selecting: Single Tap (Screen reading), Double Tap (Selection)

Custom phonebook: We developed a custom phonebook and included it into our application as shown in Figure 6(b). Our application imports the contacts from the default phonebook and shows them in a list. The gestural behavior on this contact list is similar to the dialer. There is one extra gesture available in this portion. The single tap and the double tap will work here. The single tap on every contact will read the names on the contact, and the double tap on the contact will make the call. There is another gesture in the contact list a long tap. A long tap on a contact will make that contact an emergency contact person which is another functionality of the application.

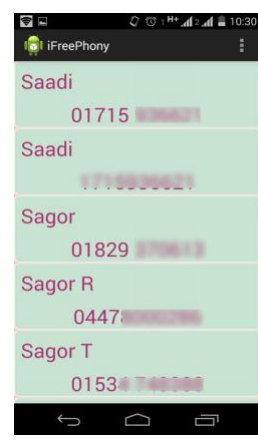

(a)

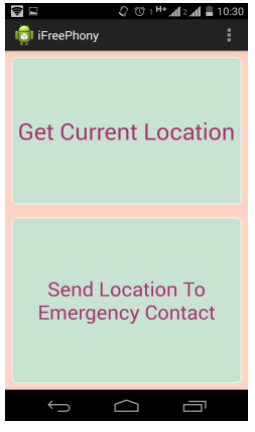

(b)

Figure 6. (a) Custom search (numbers are intentionally blurred), (b) Get help: Custom contact search method.

Search contacts: We designed an option for contact searching easier by limiting the number of contacts on the screen. We designed a sequential navigator for searching where searching is done by only entering the first letter of the contacts sequentially which are available by clicking on two navigation buttons shown in Fig. 7 as a sequence of operations. A user will first set the alphabet to jump to that cluster of contacts when the user goes 
to the phonebook. Only the letters with available contacts appear during the search.

Get help: This functionality for emergency usage is shown in Figure 7. There are two visibly large buttons in this function - the top one gets the current location of the user using the Google Location Service which is read aloud by the application; and the bottom button sends the location to an emergency contact person.

Function browsing using swipe gesture: The major problem of visuallyimpaired people is finding something. Touch screen is a very complex device. It is really tough for them to find something on the touch screen. As our application contains four different parts, we tried to make it as accessible as possible by using a special gesture called swiping. To make the functions more accessible we let the user swipe right and left to get all the functions.

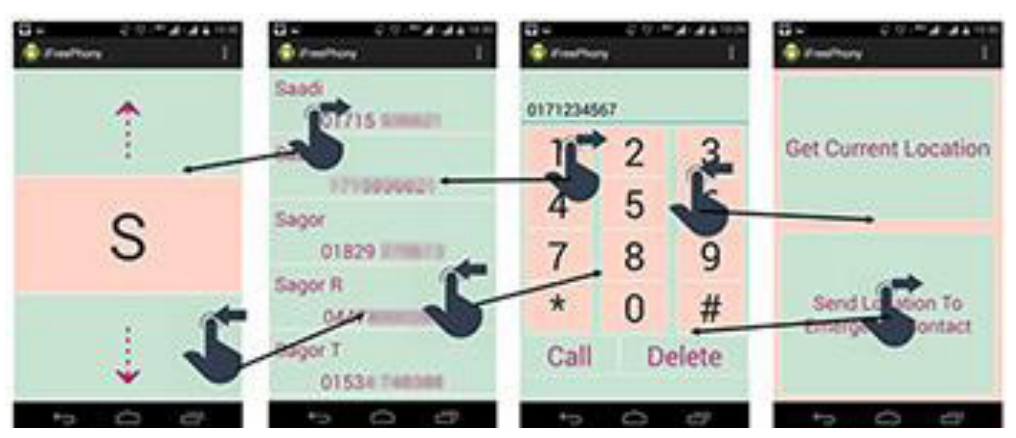

Figure 7. Browsing the functions using swipe gesture.

The default view of the application is the dialer. If a user swipes right it means that if he tries to go the left of the dialer, he will find the phonebook there. From phonebook if the user swipes right again then he will find the search or if he swipes left then he will go back to the dialer. From the dialer if the user swipes left he will find the emergency contact. Every time the user changes his position, the application will read the position of the user as can be seen in Table 1.

\section{IMPLEMENTATION}

This application is based on Direct Manipulation (Direct Manipulation, 2015) as it is developed for the Android operating system. Direct manipulation is an interaction between humans and computers which consist of continuous 
representation of objects of interest, incremental actions and feedback. We focused on these features and concentrated on how gestural interactions can be more efficient and easy for people with partial or full visual impairment as well as the elderly community requiring simple systems.

We designed this gestural event to make it easier for the VI users to access all the functions. Java Programming language was used to create this application using the Android Software Development Kit (SDK). Android Studio, the official IDE for Android development was also used to develop software. The minimum API level for this application was 4 because of some of the functionalities and libraries that were necessary for this application.

Table 1

Different Functionality Offered in system

\begin{tabular}{lll}
\hline Position & Swipe Left & Swipe Right \\
\hline Dialer & Emergency contact & Phonebook \\
Phonebook & Dialer & Search \\
Search & Phonebook & - \\
Emergency contact & - & Dialer \\
\hline
\end{tabular}

There are four main functionalities in this application. They are Dialer, Phonebook, Search and Get Help. Each function is known as Fragment. The four fragments are wrapped in a single Activity. A fragment is a modular section of an activity and the fragment's lifecycle is directly affected by the lifecycle of the host activity. The four functions are created as fragments and not activities because some of the tasks are dependent on the other tasks. Instead of destroying one activity to get to another and returning to that activity requires re-initializing the variables and objects. Instead, fragments in a single activity are used so that re-initialization of some of the objects/variables is not required. The ViewPager class of android is used in conjunction with the fragment which allows the user to flip left/right through the pages. The FragmentPagerAdapter class is implemented to generate the pages that which show that android.speech.tts is used in this application in all the fragments. Permissions that are used in the Android manifest file are shown in Figure 8.

The application is based mainly on direct manipulation as it is developed for Android OS. Direct manipulation is an interaction between humans and computers which consist of continuous representation of objects of interest, incremental actions and feedback. 


\section{<uses-permission android:name="android.permission.CALL_PHONE" $></$ uses-permission> $<$ uses-permission android:name="android.permission.READ_CONTACTS"></uses-permission> <uses-permission android:name="android.permission.SEND_SMS"></uses-permission> <uses-permission android:name="android.permission.ACCESS_FINE_LOCATION"></usespermission> <uses-permission android:name="android.permission.INTERNET"></uses-permission>}

Figure 8. Use of assistive technology on phone.

Java Programming language is used to create this application using the Android Software Development Kit (SDK). Android Studio, the official IDE for Android development is also used to develop is software. The minimum API level for this application is 4 because of some of the functionalities and libraries that are necessary for this application to run.

\section{SOLUTION APPROACH AND USER EXPERIENCES}

We conducted our study on a group of seven people who were not familiar with the system. We contacted the participants through an open call using personal contacts which mainly falls in the snowball sampling method as presented by Goodman, (1961).

We talked to seven individuals. Four of them (57\%) were female and the rest $(42 \%)$ were male. The age range of this population was from 30 to 60 years where some of the visual impairment was due to age. We had three persons with visual impairment while others were elderly people who found current smartphones difficult to use. The study asked the users to compare their access time with their existing cell phones (none of them were smart phones) with a smartphone installed with ifreePhony.

The study group shared their struggles on the smart phones candidly as:

I find it irritating to search contacts; it takes a long time for me.

I used to find it difficult to search contacts - now I save important ones using their initials.

The words are too small to read, sometimes I get frustrated.

I am fine with the current phone.

Its ok unless I am in a rush. 
The users shared similar comments on the location centric support where some users (more than 50\%) found it to be useful while the others were not very optimistic considering the Internet availability in various places.

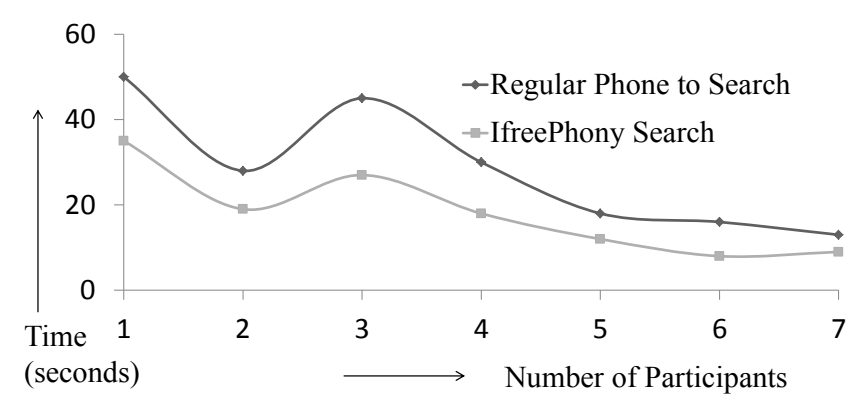

Figure 9. Searching time in comparison with and without ifreePhony.

It is evident from our study shown in Figure 9 that search time is reduced in ifreePhony compared to a regular phone search. On average the reduction is above $36 \%$. Similarly, the number of typed text increases, on average, above $57 \%$ in our system as seen in Fig. 10. We asked the users to type alphanumeric values representing names and phone numbers when we asked them to type text strings.

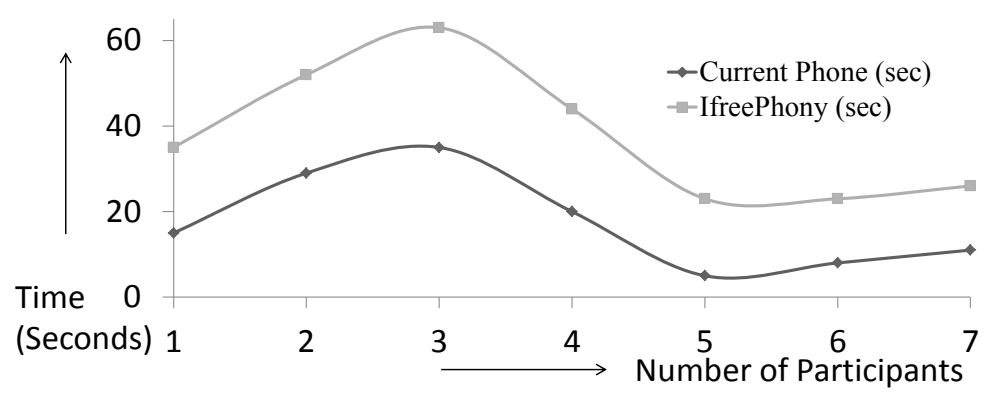

Figure 10. Typed number comparison with and without ifreePhony.

A closer look at the group of people using ifreePhony illustrates that on average there in $36.6 \%$ reduction in search time, and $57.3 \%$ increase in text typing is visible with standard deviations of 6.66 and 10.99 respectively. The percent decrease positively correlates to the increase in typed text which is evident in Figure 10. 


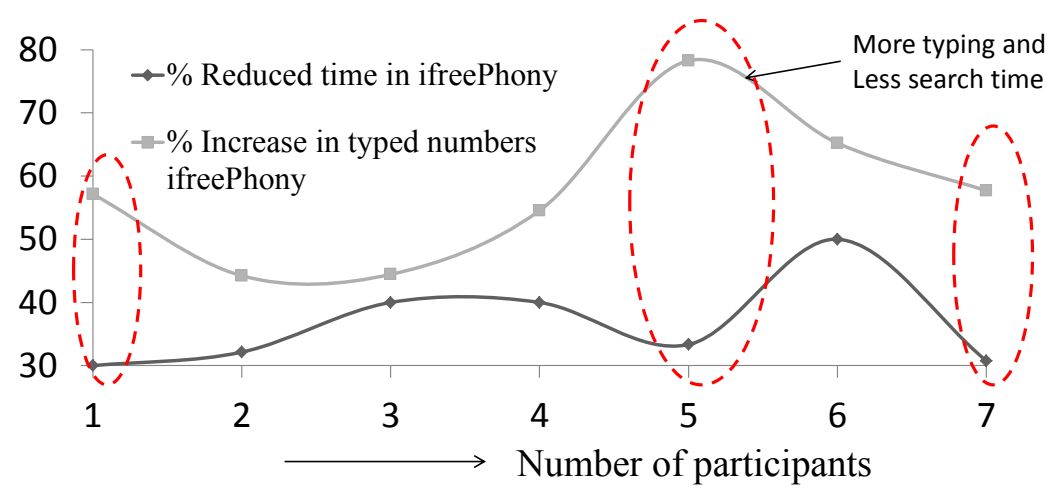

Figure 11. iFreePhony performances in terms of search time and typing text.

Grouping the participants who took relatively more reduction in search participant 1, participant 5 and participant 7 has direct correlation towards the percent increase in typed letters as 0.98 . The other group consisting of participants 2, 3, 4 and 6 correlated to 0.88 in search and percent increase in typed text. These facts are evident in Figure 11.

This correlation shows that people who are generally faster in access have an added advantage in terms of searching and typing text.

\section{CONCLUSION}

The system we have described in this paper is the reflection of the endeavor towards developing a communication assistive system which can help a big community of visually-impaired people. Mobile computing is becoming powerful and advanced day by day. Mobile phones are now so powerful that these small devices are now replacing computers. A mobile phone can do jobs which a computer can do, and because of being portable it can be used more efficiently than a computer. Also today the mobile hardware is more powerful and energy efficient. High-end devices with ultramodern sensors can perform extra-ordinary stuffs which can be used in future works to help the visually-impaired people. The current generation of mobile phones has long lasting batteries, durable touch screens and powerful processors which can run extra-ordinary assistive mobile applications. But the number of attempts to such kind of assistive technologies are not increasing at a high rate. Mobile computing is a popular topic of the current era, but most of the developers are now interested in social application development. Currently visually-impaired 
users do not benefit so much by touch-user interfaces. Our system is just a beginning of assistive technology for visually-impaired people using today's improved mobile technology. Our user study shows promises for a solution that will be able to connect the visually-impaired community through a simple yet powerful system.

\section{REFERENCES}

Ariadne, GPS. (2016). An innovative app for your mobility. Retrieved from http://www.ariadnegps.eu.

Ahmed, N., Nahar, F., Haque A. M. M., \& Chowdhury, A. M. (2015). A smart phone that acts unsmart: An assistive touch screenuser interface for visually impaired people. Proceedings of the Fourth International Conference on Internet Applications, Protocols and Services, 218-223.

Bangladesh Visually Impaired Peoples Society. (2015). Brain health: The Importance of recognizing cognitive impairment: An IAGG Consensus Conference, 731-739.

Buzzi, M. C., Buzzi, M., Leporini, B., \& Trujillo, A. (2014). Designing a text entry multimodal keypad for blind users of touchscreen mobile phones. Proceedings of the 16th International ACM SIGACCESS Conference on Computers \& Accessibility 131-136 ACM.

Dell, N., Francis, I., Sheppard, H., Simbi, R., \& Borriello, G. (2014). Field evaluation of a camera-based mobile health system in low-resource settings. Proceedings of the 16th International Conference on Humancomputer Interaction with Mobile Devices \& Services, 33-42.doi: $101145 / 2628363.2628366$.

DeRenzi, B., Borriello, G., Jackson, J., Kumar, V. S., Parikh, T. S., Virk, P., \& Lesh, N. (2011). Mobile phone tools for field-based health care workers in low-income countries. Mount Sinai Journal of Medicine: A Journal of Translational and Personalized Medicine, 78(3), 406-418.

Ghilardi, M., Junior, J., \& Manssour, I. (2016). Crosswalk localization from low resolution satellite images to assist visually impaired people. In IEEE Computer Graphics and Applications, 99. doi/10.1109/MCG.2016.50 
Goodman, L. A. (1961). Snowball sampling. The Annals of Mathematical Statistics, 148-170.

Haque, A. M., Nahar, F., \& Ahmed, N. (2014). Ifreephony: A touchscreen based user interface for people with visual impairment. Proceedings 17th International Conference on Computer and Information Technology 413-417(ICCIT), Daffodil International University, Dhaka, Bangladesh.

Hartung, C., Lerer, A., Anokwa, Y., Tseng, C., Brunette, W., \& Borriello, G. (2010). Open data kit: Tools to build information services for developing regions. Proceedings of the 4th ACM/IEEE International Conference on Information and Communication Technologies and Development, 18.

Hussain, A., Hashim, N. L., Nordin, N., \& Tahir, H. M. (2013). A metricbased evaluation model for applications on mobile phones. Journal of Information and Communication 12, 55-71.

iOS Voiceover. (2016). Retrieved from https://www.apple.com/accessibility/ ios/voiceover. Official Web Page of iOS Voiceover.

JAWS Screen Reader. (2016). Retrieved from http:/ /www. freedomscientific. com/ Products/ Blindness/ JAWS.

Kim, J. E., Bessho, M., Kobayashi, S., Koshizuka, N., \& Sakamura, K. (2016, April). Navigating visually impaired travelers in a large train station using smartphone and bluetooth low energy. Proceedings of the 31st Annual ACM Symposium on Applied Computing, 604-611.

Kvetny, E., Kahn, R., Cohen, N., \& Carmel, I. (2016). U.S. Patent No. 20,160,171,883. Washington, DC: U.S. Patent and Trademark Office.

Larson, E. C., Goel, M., Boriello, G., Heltshe, S., Rosenfeld, M., \& Patel, S. N. (2012). SpiroSmart: Using a microphone to measure lung function on a mobile phone. Proceedings of the 2012 ACM Conference on Ubiquitous Computing, 280-289.

McGookin, D., Brewster, S., \& Jiang, W. (2008). Investigating touchscreen accessibility for people with visual impairments. Proceedings of the 5th Nordic conference on Human-computer interaction: Building bridges, 298-307. 
Niazi, B., Khusro, S., Khan, A., \& Alam, I. (2016). A touch sensitive keypad layout for improved usability of smartphones for the blind and visually impaired persons. Artificial Intelligence Perspectives in Intelligent Systems, 427-436.

Oh, U., Kane, S. K., \& Findlater, L. (2013). Follow that sound: Using sonification and corrective verbal feedback to teach touchscreen gestures. Proceedings of the 15th International ACM SIGACCESS Conference on Computers and Accessibility, 13. doi: 10.1145/ 2513383.2513455.

Raposo, N., Rios, H., Lima, D., Gadelha, B., \& Castro, T. (2014). An application of mobility aids for the visually impaired. Proceedings of the 13th International Conference on Mobile and Ubiquitous Multimedia, 180-189.

Yasir, R., Nibir, M. S. I., \& Ahmed, N. (2014). A skin disease detection system for financially unstable people in developing countries. Global Science and Technology Journal, 3 (1), 77-93. 